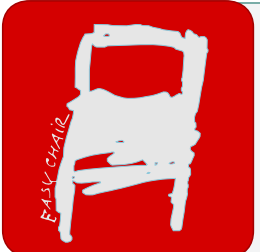

EPiC Series in Health Sciences

Volume 1, 2017, Pages 345-351

CAOS 2017. 17th Annual Meeting of the International

Society for Computer Assisted Orthopaedic Surgery

\title{
Comparison of outcome and gait analysis after robotic total knee arthroplasty between mechanical and kinematic knee alignment methods with and average 8 years follow up
}

\author{
Jong-Keun Seon, Eun-Kyoo Song, Dong-Hyun Lee, Je-Hyoung Yeo, \\ Center of Joint Disease, Chonnam National University Hwasun Hospital
}

\section{INTRODUCTION}

The achievement of optimal alignment has been reported to be an one of important factor for the success and longevity of total knee arthroplasty (TKA) [1,2]. Two basic alignment methods are used for TKA. One is the "mechanical method" introduced by Insall et al [3] and the other is the "kinematic method" introduced by Hungerford et al [4].

There were still debate of clinical results and limited previous findings detailed biomechanical properties with two alignment methods. The purpose of this study was to compare clinical outcomes and gait analysis of kinematic and kinetic parameters between two alignment methods in robotic total knee arthroplasty.

\section{MATERIALS AND METHODS}

Sixty patients were randomly assigned to undergo robotic-assisted TKA using either the mechanical (30 patients) or the kinematic (30 patients) alignment methods. All 
patients were evaluated preoperatively and at follow up visit using the Hospital Special Surgery (HSS), Western Ontario and McMaster Universities (WOMAC) scoring systems, range of motion (ROM) assessment, and Knee Society (KS) pain and function score. Radiographic evaluations were performed preoperatively and postoperatively using standing full-leg radiographs to determine overall coronal plane alignment and plane radiograph to measure component alignment in the sagittal and coronal plane.

Ten age and gender matched patients of each group underwent gait analysis at minimum 5-years postoperatively. We evaluated parameters including knee varus moment and knee varus forces, and find out the difference between two groups.

\section{RESULTS}

The mean follow up duration of both groups was 8.1 years (mechanical method) and 8.0 years (kinematic methods). Clinical outcomes between two groups showed no significant difference in HSS, WOMAC, ROM, KS pain and function score at the last follow up (Table 1). There were no significant difference in varus and valgus laxity assessment, mechanical alignment of the lower limb, and perioperative complications (Table 2).

In gait analysis, there were no significant difference in kinematic and kinetic parameters including varus force and varus moment (Figure1)(Table 3). However, significant difference was observed in the mediolateral ground reaction force $(P=0.01)$

\section{DISCUSSION}

The goal of primary total knee arthroplasty is to reestablish a normal mechanical axis using a well-fixed stable prosthesis [2,5]. Historically, total knee alignment methods have been classified as mechanical or kinematic alignment methods. For mechanical alignment methods, which are the most commonly used, the goal is to establish a joint line perpendicular to the mechanical axis. However, it should be remembered that $1 \mathrm{~mm}$ mediolateral placement errors of a femoral head will lead to $3^{\circ}-4.5^{\circ}$ errors in the 
orientation of the femoral cut [6].

Hungerford et al. proposed the kinematic alignment method because it mimics the normal anatomical characteristics of knee and produces better kinematics. However, it usually has excessive varus may result because the correct assessment of $3^{\circ}$ of varus is difficult to achieve, and this could lead to overall varus malalignment of the lower limb, which is deleterious for prosthesis survival and mechanical function [7].

In this study, we found that the both of mechanical and kinematic alignment methods provide comparable and successful clinical and radiographic outcomes after robotic TKA and there were no functional difference between two alignment methods during walking.

\section{REFERENCES}

1. Bargren JH, Blaha JD, Freeman MAR. Alignment in total knee arthroplasty: correlated biomechanical and clinical observations. Clin Orthop 1983;173:178.

2. Tew $\mathrm{M}$, Waugh $\mathbf{W}$. Tibiofemoral alignment and the results of knee replacement. J Bone Joint Surg Br 1985;67B:551.

3. Insall J, Scott WN, Ranawat CS. The total condylar knee prosthesis: a report of two hundred and twenty cases. J Bone Joint Surg Am 1979;61A:173.

4. Hungerford DS, Kenna RV, Krackow KA. The porous-coated anatomic total knee. Orthop Clin North Am 1982;13:103.

5. Jeffery RS, Morris RW, Denham RA. Coronal alignment after total knee replacement. J Bone Joint Surg Br 1991;73B:709.

6. Teter KE, Bregman D, Colwell CW. The efficacy of intramedullary femoral alignment in total knee replacement. Clin Orthop 1995;321:117.

7. Lotke PA, Ecker ML. Influence of positioning of prosthesis in total knee replacement. J Bone Joint Surg Am 1977;59A:77. 


\section{DISCLOSRES}

This research did not receive any specific grant from funding agencies in the public, commercial, or not-for-profit sectors. 


\section{FIGURE}

Figure 1. Gait analysis. Yellow line indicates mechanical group and blue line indicates kinematic group.
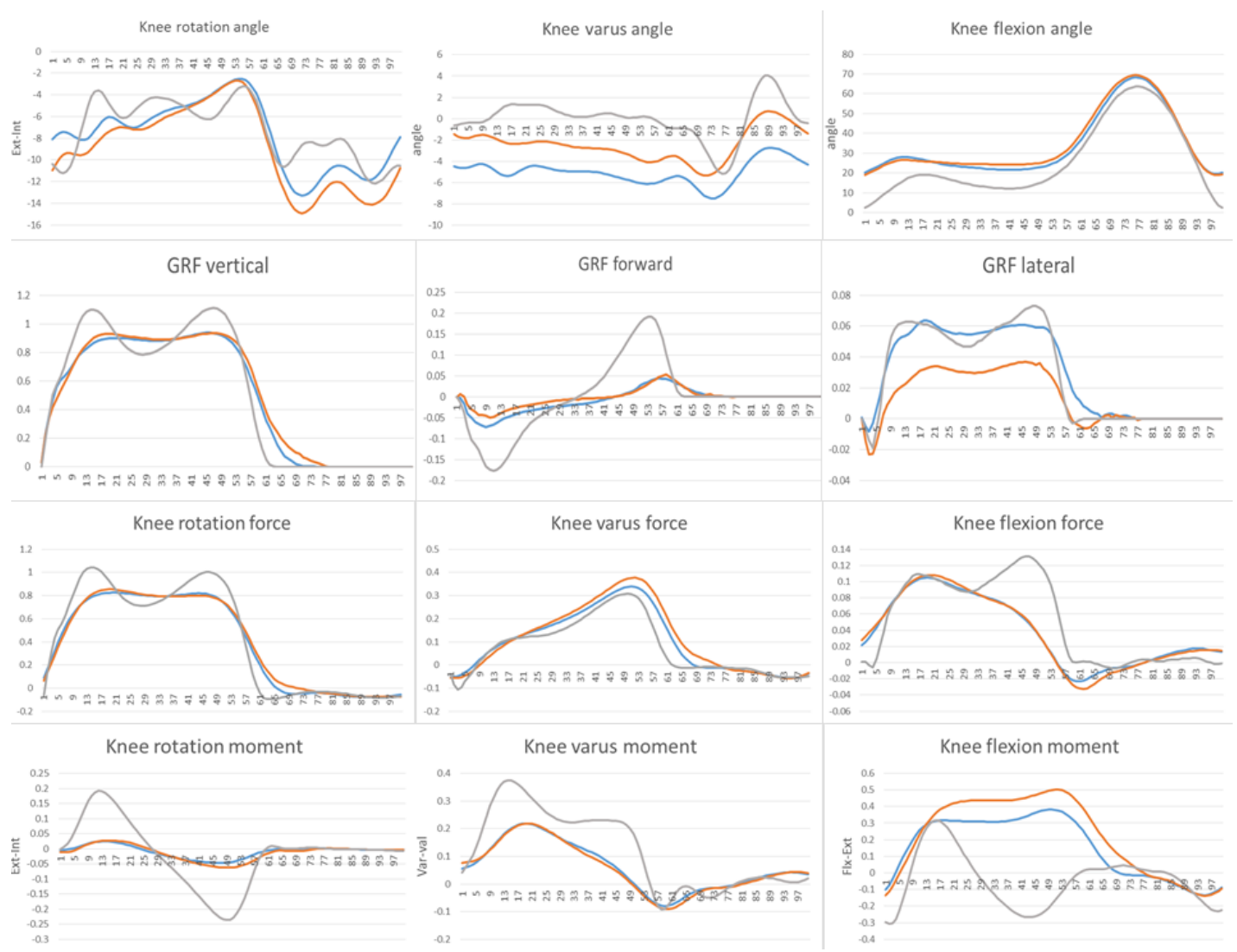


\section{TABLES}

Table 1. Clinical outcomes between two alignment methods at the final follow up

\begin{tabular}{|l|c|c|c|}
\hline & Mechanical group $(\mathrm{N}=30)$ & Kinematic group $(\mathrm{N}=30)$ & $P$-value \\
\hline HSS & $94.8 \pm 5.5$ & $93.2 \pm 8.0$ & 0.81 \\
\hline WOMAC & $20.4 \pm 1.8$ & $19.3 \pm 1.9$ & 0.56 \\
\hline ROM & $129.0 \pm 11.5$ & $125.0 \pm 11.5$ & 0.83 \\
\hline KSS (pain) & $47.2 \pm 7.5$ & $47.5 \pm 5.6$ & 0.19 \\
\hline KSS (function) & $93.0 \pm 9.1$ & $90.1 \pm 10.5$ & 0.72 \\
\hline
\end{tabular}

Table 2. Radiographic outcomes between two alignment methods at the final follow up

\begin{tabular}{|l|c|c|c|}
\hline & $\begin{array}{c}\text { Mechanical group } \\
(\mathrm{N}=30)\end{array}$ & $\begin{array}{c}\text { Kinematic group } \\
(\mathrm{N}=30)\end{array}$ & $P$-value \\
\hline Mechanical axis & $-0.7 \pm 1.7$ & $-0.4 \pm 2.0$ & 0.76 \\
\hline Coronal femoral inclination & $89.5 \pm 0.4$ & $91.7 \pm 1.9$ & 0.54 \\
\hline Coronal tibial inclination & $90.1 \pm 0.4$ & $87.5 \pm 1.7$ & 0.42 \\
\hline Sagittal femoral inclination & $3.0 \pm 1.5$ & $3.8 \pm 2.7$ & 0.31 \\
\hline Sagittal tibial inclination & $83.6 \pm 1.0$ & $82.5 \pm 2.8$ & 0.20 \\
\hline Mean varus laxity & $68 \pm 3.3$ & $3.5 \pm 2.6$ & 0.16 \\
\hline Mean valgus laxity & $5.8 \pm 3.3$ & $3.2 \pm 2.5$ & 0.49 \\
\hline Total joint laxity & $10.3 \pm 1.0$ & $9.1 \pm 2.8$ & 0.18 \\
\hline
\end{tabular}


Table 3. Spatiotemporal results in gait analysis

\begin{tabular}{|l|c|c|c|}
\hline & Mechanical group (N=30) & Kinematic group (N=30) & $P$-value \\
\hline $\begin{array}{l}\text { Cadence } \\
\text { (steps/min) }\end{array}$ & $109.0 \pm 14.9$ & $106.9 \pm 17.5$ & 0.49 \\
\hline $\begin{array}{l}\text { Total support (\% } \\
\text { cycle) }\end{array}$ & $63.2 \pm 3.4$ & $62.7 \pm 3.1$ & 0.63 \\
\hline Stride length (cm) & $89.6 \pm 17.8$ & $80.9 \pm 31.6$ & 0.68 \\
\hline Stride time (sec) & $1.1 \pm 0.2$ & $1.2 \pm 0.2$ & 0.22 \\
\hline $\begin{array}{l}\text { Gait velocity } \\
(\mathrm{cm} / \mathrm{s})\end{array}$ & $82.0 \pm 21.8$ & $79.7 \pm 23.6$ & 0.28 \\
\hline
\end{tabular}

